\title{
Projeto de educação em saúde sobre Sífilis durante a pandemia de COVID-19 por meio de redes sociais: $U m$ relato de experiência
}

\author{
Health Education Project on Syphilis in the COVID-19 pandemic through social media: An
} experience report

Proyecto de educación en salud sobre la Sífilis durante la pandemia de COVID-19 por medio de redes sociales: Un informe de experiencia

\author{
João Victor Coimbra França \\ ORCID: https://orcid.org/0000-0003-4210-4230 \\ Universidade Estadual do Piauí, Brasil \\ E-mail: victor.joao12@outlook.com \\ Isabel Bacelar Fontenele Araújo \\ ORCID: https://orcid.org/0000-0003-4448-8987 \\ Universidade Estadual do Piauí, Brasil \\ E-mail: isabelaraujo@aluno.uespi.br \\ Luis Felipe Rodrigues Brandão de Barros \\ ORCID: https://orcid.org/0000-0002-1690-6638 \\ Universidade Estadual do Piauí, Brasil \\ E-mail: luisbarros@aluno.uespi.br \\ Luiz Fernando Reis Camboim \\ ORCID: https://orcid.org/0000-0001-5261-5494 \\ Universidade Estadual do Piaú, Brasil \\ E-mail: luizcamboim@aluno.uespi.br \\ Rafael Everton Assunção Ribeiro da Costa \\ ORCID: https://orcid.org/0000-0002-0798-890X \\ Universidade Estadual do Piauí, Brasil \\ E-mail: rafaelcosta@aluno.uespi.br \\ Antônio Luiz Martins Maia Filho \\ ORCID: https://orcid.org/0000-0001-6184-8003 \\ Universidade Estadual do Piauí, Brasil \\ E-mail: almmaiaf@ccs.uespi.br \\ Samylla Miranda Monte \\ ORCID: https://orcid.org/0000-0003-1329-9825 \\ Universidade Estadual do Piauí, Brasil \\ E-mail: samyllamonte@ccs.uespi.br \\ Rosemarie Brandim Marques \\ ORCID: https://orcid.org/0000-0002-4792-7407 \\ Universidade Estadual do Piauí, Brasil \\ E-mail: rosebmarques@ccs.uespi.br
}

\begin{abstract}
Resumo
Introdução: As infecções sexualmente transmissíveis (ISTs) são um importante problema de saúde pública, trazendo muitas consequências para a população mundial. A sífilis é uma IST causada pela bactéria Treponema pallidum e constitui uma importante doença no contexto da saúde brasileira pelo aumento de casos nos últimos anos. Objetivo: Descrever as ações remotas em redes sociais de um projeto de educação em saúde sobre sífilis, desenvolvido durante a pandemia de COVID-19, e analisar seus impactos na comunidade. Metodologia: O presente projeto utilizou a plataforma digital Instagram ${ }^{\circledR}$ para divulgar panfletos sobre a doença, tirar dúvidas da população alvo, além de coletar dados dos usuários da rede social sobre o tema. Resultados: Os resultados foram quantificados de acordo com as visualizações das postagens veiculadas. O panfleto inicial sobre sífilis obteve um alcance de 2513 visualizações e, nas semanas de postagem posteriores, houve os totais de 7847, 1037, 1058, 3793 e 2481 visualizações em cada tópico, respectivamente. Considerações Finais: Cabe destacar que, inicialmente, o objetivo do projeto de extensão universitária era ser presencial, tanto com a utilização de testes rápidos para detecção de sífilis como com a apresentação de palestras em uma comunidade carente de Teresina, Piauí, Brasil. No entanto, o projeto teve que ser adaptado ao meio digital devido às medidas relacionadas à pandemia de COVID-19 recomendadas por autoridades de saúde. Ainda, apesar da impossibilidade de seguir o planejamento inicial, o projeto atingiu um bom alcance, inclusive sendo divulgado nas plataformas online da Universidade Estadual do Piauí.
\end{abstract}

Palavras-chave: Sífilis; Educação em saúde; COVID-19. 


\begin{abstract}
Introduction: Sexually Transmitted Infections (STIs) are major public health issues, bringing many consequences to the world population. Syphilis is an STI caused by the Treponema pallidum bactéria and constitutes a substantial disease of the Brazilian health scenery, due to the increase of cases in past years. Objective: To describe the remote activities on social media of a health education project about syphilis, developed during the COVID-19 pandemic, and analyzing its impacts on the community. Methodology: The current project used the Instagram ${ }^{\circledR}$ digital platform to divulge fliers concerning the disease, answer the target audience's questions, and also collect data on the theme from social media users. Results: The results were quantified according to the weekly post views. The initial flier on Syphilis accomplished a total reach of 2513 views and, in the subsequent posting weeks, there were totals of 7847, 1037, 1058, 3793, and 2481 views on each topic, respectively. Final Considerations: It is noteworthy that, initially, the university extension project was supposed to be presential, not only using rapid syphilis detection tests but also presenting lectures to a necessitous community in Teresina, Piauí, Brazil. However, the activity had to be adapted to digital means, on account of measures related to the COVID-19 pandemic recommended by health authorities. Still, despite the impossibility to carry on the initial planning, the project achieved a satisfactory reach, to the point of even being divulged by the online platforms of the State University of Piauí.
\end{abstract}

Keywords: Syphilis; Health education; COVID-19.

\title{
Resumen
}

Introducción: Las infecciones de transmisión sexual (ITS) son un importante problema de salud pública, con muchas consecuencias para la población mundial. La sífilis es una ITS causada por la bacteria Treponema pallidum y constituye una enfermedad importante en el contexto de la salud brasileña debido al aumento de casos en los últimos años. Objetivo: Describir las acciones remotas en redes sociales de un proyecto de educación en salud sobre sífilis, desarrollado durante la pandemia COVID-19, y analizar sus impactos en la comunidad. Metodología: Este proyecto utilizó la plataforma digital Instagram ${ }^{\circledR}$ para difundir folletos sobre la enfermedad, responder preguntas de la población objetivo y recolectar datos de los usuarios de las redes sociales sobre el tema. Resultados: Los resultados se cuantificaron según las opiniones de las publicaciones publicadas. El panfleto inicial sobre sífilis alcanzó las 2513 visitas y, en las siguientes semanas de publicación, hubo un total de 7847, 1037, 1058, 3793 y 2481 visitas sobre cada tema, respectivamente. Consideraciones finales: Cabe señalar que, inicialmente, el objetivo del proyecto de extensión universitaria era ser presencial, tanto con el uso de pruebas rápidas para la detección de sífilis como con la presentación de conferencias en una comunidad pobre de Teresina, Piaú, Brasil. Sin embargo, el proyecto tuvo que ser adaptado al medio digital debido a las medidas relacionadas con la pandemia COVID-19 recomendadas por las autoridades sanitarias. Aun así, a pesar de la imposibilidad de seguir la planificación inicial, el proyecto alcanzó un buen alcance, incluso se difundió en las plataformas en línea de la Universidad Estatal de Piauí.

Palabras clave: Sífilis; Educación en salud; COVID-19.

\section{Introdução}

As Infecções Sexualmente Transmissíveis (IST) se destacam mundialmente como um importante problema de saúde pública, sobretudo nos países em desenvolvimento, trazendo uma série de consequências sanitárias, sociais e econômicas (Pinto, Basso, Barros \& Gutierrez, 2018). Cabe destacar que dificuldades para o acesso a serviços de saúde de qualidade ainda são grandes obstáculos no enfrentamento destas doenças, sobretudo em grupos sociais marginalizados (Pinto et al., 2018).

A sífilis é uma IST causada pela bactéria Treponema pallidum, curável e exclusiva da raça humana, que se apresenta em variadas manifestações clínicas e em diferentes estágios (sífilis primária, secundária, latente e terciária), com maior risco de transmissão nas classificações primária e secundária (Peeling et al., 2018; Ministério da Saúde (MS), 2017). A sífilis pode ser transmitida durante relações sexuais sem uso de preservativo ou verticalmente durante a gestação e/ou o parto (MS, 2017).

Rowley et al. (2019) estimam a ocorrência de cerca de 1 (um) milhão de casos de IST por dia, entre clamídia, gonorreia, sífilis e tricomoníase. A presença de uma IST, como a sífilis ou gonorreia, também aumenta consideravelmente o risco de adquirir ou transmitir a infecção pelo vírus HIV (MS, 2017).

Dados divulgados pelo Ministério da Saúde apontam um aumento no número de casos de sífilis no Brasil em todos os cenários da infecção (MS, 2018). Em comparação ao ano de 2016, observou-se um aumento de 28,5\% na taxa de detecção em gestantes, 16,4\% na incidência de sífilis congênita e 31,8\% na incidência de sífilis adquirida (MS, 2018). A taxa de detecção da sífilis adquirida no Brasil passou de 44,1/100 mil habitantes em 2016 para 58,1 casos para cada 100 mil habitantes em 2017 (MS, 2018). 
O Piauí, entre os anos de 2010 e 2017, apresentou a menor taxa de detecção (por 100.000 habitantes) de sífilis adquirida, apresentando um valor de 10,7 casos/100 mil habitantes neste período (MS, 2018). Quanto à sífilis congênita, no período de 2007 a 2017, o estado apresentou 8,8 casos/1.000 nascidos vivos, e, neste mesmo período, apresentou um coeficiente de mortalidade infantil por sífilis congênita de 17/100 mil nascidos vivos (MS, 2018).

A exposição das pessoas ao adoecimento pode resultar não somente de um conjunto de aspectos individuais, mas de fatores coletivos e contextuais que acarretam aos indivíduos maior suscetibilidade frente à disponibilidade de recursos para se protegerem contra a sífilis ou outras infecções (Ayres, Freitas, Santos, Saletti Filho, \& França Júnior, 2003). Diante do exposto, este estudo buscou descrever as ações remotas em redes sociais de um projeto de educação em saúde sobre sífilis desenvolvido durante a pandemia de COVID-19 e analisar os seus impactos na comunidade.

\section{Metodologia}

Trata-se de um estudo qualitativo, no formato de relato de experiência, tendo como lócus de vivência o projeto "Levantamento do perfil epidemiológico e socioeconômico de sífilis em adolescentes e adultos de uma comunidade carente em Teresina-Piauí", vinculado ao Programa Institucional de Bolsas de Extensão Universitária (PIBEU) da Universidade Estadual do Piauí (UESPI). A metodologia utilizada possui respaldo na literatura científica, sendo realizada em outras regiões do país de forma similar (Rodrigues, Freitas, \& Henriques, 2020; Dantas-Silva, Brito, Araújo, \& Macedo, 2020).

Haja vista as intempéries da pandemia do COVID-19, fez-se necessário desenvolver novas metodologias para atingir a população e dar continuidade ao projeto de educação em saúde sobre sífilis. Considerou-se como alternativa o uso da rede social Instagram® para a veiculação de informações educativas, além do esclarecimento de possíveis dúvidas, para promover um elo com a população.

Desse modo, organizou-se, inicialmente, a postagem de um panfleto, seguido, algum tempo após, de um cronograma temático com duração de cinco semanas. A cada semana, divulgou-se o material próprio, desenvolvido a partir das temáticas selecionadas e adaptados à realidade do público-alvo das redes sociais. Evidenciou-se a postagem em dias e horários estratégicos, na rede social Instagram ${ }^{\circledR}$, por meio de cinco contas pessoais distintas, para maior alcance de usuários interagindo com o conteúdo. Utilizou-se a modalidade de postagem "story", explorando alguns recursos disponíveis, como "enquetes" e "perguntas".

Passadas cerca de $24 \mathrm{~h}$ da postagem inicial, as visualizações foram registradas por meio de capturas de tela por cada integrante, em sua conta, e então os dados foram extraídos, tabulados e analisados, utilizando a ferramenta virtual QuickCalcs (Graphpad, 2021).

\section{Resultados}

A partir do exposto, os resultados foram quantificados de acordo com o número de visualizações das postagens semanais veiculadas a partir de cada uma das contas pessoais utilizadas na rede social Instagram. De acordo com a sistematização proposta para as atividades, foram publicados: Panfleto Informativo sobre Sífilis com alcance total de 2.513 visualizações (Tabela 1) e, posteriormente, a sequência de postagens semanais. 
Tabela 1. Visualizações do panfleto informativo sobre sífilis.

\begin{tabular}{ccccccc}
\hline Participante & Post 1 & Post 2 & Post 3 & Média & DP & Total \\
\hline Conta 1 & 159 & 150 & 142 & 150,33 & 8,5 & 451 \\
Conta 2 & 232 & 221 & 212 & 221,67 & 10,02 & 665 \\
Conta 3 & 126 & 121 & 116 & 121,00 & 5,00 & 363 \\
Conta 4 & 249 & 238 & 236 & 231,00 & 7,00 & 723 \\
Conta 5 & 111 & 105 & 95 & 103,67 & 8,08 & 311 \\
\hline Total & 877 & 835 & 801 & 837,67 & 38,07 & 2513 \\
\hline
\end{tabular}

Legenda: DP - Desvio Padrão. Destacam-se, nessa tabela, a média e o total de visualizações. Fonte: Autores (2020).

Na primeira semana, publicaram-se postagens de enquetes sobre hábitos de higiene pessoal, e como resultado, houve um total de 7.847 visualizações (Tabela 2).

Tabela 2. Respostas às postagens interativas da primeira semana da segunda rodada de postagens.

\begin{tabular}{ccccccc}
\hline Participante & Conta 1 & Conta 2 & Conta 3 & Conta 4 & Conta 5 & Total \\
\hline Post 1 & 220 & 149 & 91 & 153 & 142 & 755 \\
Post 2 & 217 & 146 & 92 & 149 & 129 & 733 \\
Post 3 & 219 & 150 & 94 & 149 & 121 & 733 \\
Post 4 & 219 & 143 & 89 & 149 & 98 & 698 \\
Post 5 & 210 & 147 & 93 & 145 & 96 & 691 \\
Post 6 & 217 & 145 & 93 & 145 & 101 & 701 \\
Post 7 & 216 & 146 & 92 & 147 & 115 & 716 \\
Post 8 & 217 & 147 & 92 & 147 & 110 & 713 \\
Post 9 & 218 & 147 & 90 & 146 & 106 & 707 \\
Post 10 & 213 & 147 & 90 & 144 & 104 & 698 \\
Post 11 & 218 & 147 & 90 & 144 & 103 & 702 \\
\hline Média & 216,73 & 146,73 & 91,45 & 147,09 & 111,36 & 713,36 \\
DP & 2,90 & 1,85 & 1,57 & 2,74 & 14,26 & 19,51 \\
Total & 2384 & 1614 & 1006 & 1618 & 1225 & 7847
\end{tabular}

Legenda: DP - Desvio Padrão. Destacam-se, nessa tabela, tanto a grande média como a considerável quantidade total de visualizações. Fonte: Autores (2020).

Quanto aos questionamentos, $97,60 \%$ das respostas afirmaram que tomam banhos todos os dias (DP: 2,07); 98,00\% lavam as regiões íntimas em todos os banhos (DP: 1,22); 93,60\% relataram que lavam os cabelos ao menos 2 vezes por semana (DP: 2,88); 99,20\% usam sabonete ou sabão no banho (DP: 1,10); 98,40\% usam shampoo ao lavar os cabelos (DP: 1,52); 84,20\% usam condicionador (DP: 6,72); 68,40\% fazem a manutenção das unhas pelo menos 2 vezes por mês (DP: 12,42); 40,80\% têm o hábito de roer as unhas (DP: 2,59); 65,60\% sempre lavam as mãos antes das refeições (DP: 5,86); 93,80\% escovam os dentes ao menos 2 vezes por dia (DP: 2,86) e 97,40\% mudam de roupa íntima todos os dias (DP: 1,14) (Tabela 3). 
Tabela 3. Porcentagem de respostas "sim" em cada pergunta.

\begin{tabular}{cccccccc}
\hline Participante & Cont 1 & Cont 2 & Cont 3 & Cont 4 & Cont 5 & Média & DP \\
\hline Post 1 & $99,00 \%$ & $98,00 \%$ & $99,00 \%$ & $98,00 \%$ & $94,00 \%$ & $97,60 \%$ & 2,07 \\
Post 2 & $99,00 \%$ & $98,00 \%$ & $98,00 \%$ & $99,00 \%$ & $96,00 \%$ & $98,00 \%$ & 1,22 \\
Post 3 & $95,00 \%$ & $96,00 \%$ & $90,00 \%$ & $91,00 \%$ & $96,00 \%$ & $93,60 \%$ & 2,88 \\
Post 4 & $100 \%$ & $100 \%$ & $98,00 \%$ & $100 \%$ & $98,00 \%$ & $99,20 \%$ & 1,10 \\
Post 5 & $100 \%$ & $99,00 \%$ & $96,00 \%$ & $99,00 \%$ & $98,00 \%$ & $98,40 \%$ & 1,52 \\
Post 6 & $89,00 \%$ & $83,00 \%$ & $87,00 \%$ & $89,00 \%$ & $73,00 \%$ & $84,20 \%$ & 6,72 \\
Post 7 & $52,00 \%$ & $71,00 \%$ & $60,00 \%$ & $76,00 \%$ & $83,00 \%$ & $68,40 \%$ & 12,42 \\
Post 8 & $38,00 \%$ & $44,00 \%$ & $40,00 \%$ & $39,00 \%$ & $43,00 \%$ & $40,80 \%$ & 2,59 \\
Post 9 & $62,00 \%$ & $69,00 \%$ & $57,00 \%$ & $70,00 \%$ & $70,00 \%$ & $65,60 \%$ & 5,86 \\
Post 10 & $96,00 \%$ & $94,00 \%$ & $89,00 \%$ & $94,00 \%$ & $96,00 \%$ & $93,80 \%$ & 2,86 \\
Post 11 & $97,00 \%$ & $97,00 \%$ & $98,00 \%$ & $99,00 \%$ & $96,00 \%$ & $97,40 \%$ & 1,14 \\
\hline
\end{tabular}

Legenda: Cont - Conta; DP - Desvio Padrão. Das médias de porcentagens observadas, destacam-se os 68,40\% que fazem a manutenção das unhas pelo menos 2 vezes por mês, no post 7; os 40,80\% que têm o hábito de roer as unhas, no post 8 , e os $65,60 \%$ que sempre lavam as mãos antes das refeições, no post 9. Fonte: Autores (2020).

Na segunda semana, publicou-se um informativo sobre contração de sífilis, houve um total de 1.037 visualizações (Tabela 4); na terceira semana, publicou-se um informativo sobre sinais e sintomas de sífilis e houve um total de 1.058 visualizações (Tabela 5); na quarta semana, publicou-se um informativo sobre o tratamento da sífilis e houve um total de 3.793 visualizações (Tabela 6); na quinta semana, publicou-se um informativo sobre prevenção e complicações da sífilis, com 2.481 visualizações (Tabela 7).

Tabela 4. Visualizações da segunda semana da segunda rodada de postagens.

\begin{tabular}{ccccccc}
\hline Participante & Post 1 & Post 2 & Post 3 & Média & DP & Total \\
\hline Conta 1 & 182 & 174 & 167 & 174,33 & 7,51 & 523 \\
Conta 2 & $*$ & $*$ & $*$ & $*$ & $*$ & $*$ \\
Conta 3 & $*$ & $*$ & $*$ & $*$ & $*$ & $*$ \\
Conta 4 & 180 & 171 & 163 & 171,33 & 8,50 & 514 \\
Conta 5 & $*$ & $*$ & $*$ & $*$ & $*$ & $*$ \\
\hline Total & 362 & 345 & 330 & 345,67 & 16,01 & 1037
\end{tabular}

Legenda: *Integrante esqueceu de registrar as visualizações dentro de 48h após a postagem; DP - Desvio Padrão. Destacam-se, na tabela, a média e o total de visualizações. Fonte: Autores (2020). 
Tabela 5. Visualizações da terceira semana da segunda rodada de postagens.

\begin{tabular}{ccccccc}
\hline Participante & Post 1 & Post 2 & Post 3 & Média & DP & Total \\
\hline Conta 1 & 134 & 125 & 121 & 126,67 & 6,66 & 380 \\
Conta 2 & 120 & 111 & 103 & 111,33 & 8,50 & 334 \\
Conta 3 & 69 & 67 & 59 & 65,00 & 5,29 & 195 \\
Conta 4 & $*$ & $*$ & $*$ & $*$ & $*$ & $*$ \\
Conta 5 & 50 & 50 & 49 & 49,67 & 0,58 & 149 \\
\hline Total & 373 & 353 & 332 & 352,67 & 20,50 & 1058
\end{tabular}

Legenda: *Participante esqueceu de registrar as visualizações dentro de 48h após a postagem; DP - Desvio Padrão. Destacam-se, na tabela, a média e o total de visualizações. Fonte: Autores (2020).

Tabela 6. Visualizações da quarta semana da segunda rodada de postagens.

\begin{tabular}{cccccccccccc}
\hline Participante & Post 1 & Post 2 & Post 3 & Post 4 & Post 5 & Post 6 & Post 7 & Post 8 & Post 9 & DP & Total \\
\hline Conta 1 & 103 & 100 & 100 & 97 & 88 & 85 & 82 & 82 & 92,13 & 8,77 & 737 \\
Conta 2 & 137 & 129 & 125 & $*$ & 115 & 98 & 97 & 94 & 113,57 & 17,42 & 795 \\
Conta 3 & 136 & 130 & 127 & 122 & 116 & 113 & 110 & 109 & 120,38 & 9,96 & 963 \\
Conta 4 & 122 & 117 & 114 & 108 & 102 & 100 & 97 & 97 & 107,13 & 9,63 & 857 \\
Conta 5 & 64 & 60 & 59 & 54 & 53 & 51 & 50 & 50 & 55,13 & 5,25 & 441 \\
\hline Total & 562 & 536 & 525 & 381 & 474 & 447 & 436 & 432 & 474,13 & 61,85 & 3793
\end{tabular}

Legenda: *: Integrante esqueceu-se de postar. Destacam-se, na tabela, o notável total de visualizações. Fonte: Autores (2020).

Tabela 7. Visualizações da quinta semana da segunda rodada de postagens.

\begin{tabular}{cccccccccc}
\hline Participante & Post 1 & Post 2 & Post 3 & Post 4 & Post 5 & Post 6 & Média & DP & Total \\
\hline Conta 1 & 126 & 117 & 111 & 109 & 105 & 103 & 111,83 & 8,50 & 671 \\
Conta 2 & 129 & 108 & 95 & 88 & 82 & 76 & 96,33 & 19,46 & 578 \\
Conta 3 & 77 & 66 & 59 & 56 & 56 & 55 & 61,50 & 8,60 & 369 \\
Conta 4 & 123 & 113 & 108 & 101 & 96 & 96 & 106,17 & 10,65 & 637 \\
Conta 5 & 45 & 41 & 40 & 36 & 32 & 32 & 37,67 & 5,24 & 226 \\
\hline Total & 500 & 445 & 413 & 390 & 371 & 362 & 413,50 & 51,93 & 2481
\end{tabular}

Legenda: DP - Desvio Padrão. Destacam-se, na tabela, a média e o total de visualizações. Fonte: Autores (2020).

\section{Discussão}

A universidade é construída pelo tripé Ensino, Pesquisa e Extensão, sendo esta última orientada no sentido de levar o conhecimento produzido pelas instituições de ensino para além dos seus muros, relacionando-se com a comunidade em que se está inserida, possibilitando a práxis dos conhecimentos construído por essas instituições e, em simultâneo, a formação de novos saberes com a comunidade (Pinotti, 2020). Dessa forma, há uma relação dialética entre os extensionistas e a população beneficiária desses projetos, em que os aprendizados são disseminados em uma via de mão dupla entre estes e os seus atores (Diniz et al., 2020). 
A prática da extensão universitária é reconhecida por diversos autores que demonstram os benefícios para o futuro profissional dos estudantes extensionistas, como aquisição de pluralidades de pensamentos e a aplicação dos conhecimentos apreendidos em sala de aula e desenvolvimento de competências para o futuro profissional (Moura et al, 2012; Oliveira \& Almeida Júnior, 2015; Chung et al, 2020); a melhora do trabalho em equipe, incluindo a prática em equipes multiprofissionais e o trato com coletivo (Resende, Alves, Coutinho , Bragagnoli, \& Araújo, 2013; Ferraz \& Del Ciampo, 2019); assim como a formação humanista do estudante, a sua vivência em situações distintas a sua realidade e o seu amadurecimento profissional (Luna et al, 2020; Moraes et al, 2016). Isso evidencia a importância da participação de projetos de extensão para criar profissionais mais preparados para os desafios reais no mercado de trabalho e das demandas sociais.

Com a pandemia do COVID-19, a atividade extensionista recebe outros desafios em que a mesma deve-se integrar à comunidade na época em que a regra é o distanciamento social, assim como, paralelamente, a sociedade fica mais vulnerável com a crise sanitária e econômica, o que possibilitou que a extensão universitária agisse em diversas frentes, desenvolvendo novos mecanismos de atuação em consonância aos protocolos de segurança, tais como criação de Equipamentos de Proteção Individual, Webinários e criação de cartilhas educativas online (Diniz et al, 2020; Silva et al, 2020).

Inicialmente, este projeto estava voltado à aplicação de testes rápidos numa população da periferia da cidade de Teresina-PI, juntamente a atividades presenciais de educação em saúde. Assim, de janeiro a março, os participantes planejaram e discutiram os aspectos da ação, tais como a logística, os gastos, a abordagem aos entrevistados e a organização entre os integrantes do projeto. No entanto, devido à proibição de atividades presenciais determinadas, inicialmente pelo Decreto Municipal $n^{\circ} 19.531$ (2020), os integrantes decidiram suspender as atividades por tempo indeterminado, até que houvesse um parecer mais favorável do governo quanto às atividades presenciais.

Porém, sem uma previsão próxima de encerramento da quarentena, decidiu-se, em julho, converter o projeto a uma tática virtual de educação em saúde, juntamente a uma análise epidemiológica da sífilis em Teresina. O uso de redes sociais para perpetuação do projeto de extensão valida-se nos seguintes fatos: a rede social escolhida, o Instagram®, é uma das principais ferramentas deste intuito utilizada por empresas não só do Brasil, mas também do mundo; ademais, facilita o compartilhamento da divulgação científica, tem representatividade e grande influência nas atuais relações sociais (Rocha et al., 2020). Nesse momento, também se iniciou o planejamento e a divisão de trabalho quanto ao novo projeto.

No mês seguinte, com a divulgação do panfleto virtual, os resultados foram satisfatórios, com alcance notável. Além disso, neste mesmo mês, também houve a menção do projeto no próprio site da Universidade Estadual do Piauí, fato que alavancou a visibilidade do projeto (Fernandes, 2020). Em outubro, visto que persistia a quarentena, os integrantes decidiram realizar mais postagens, de caráter mais informal, a fim de atingir ainda mais indivíduos com o projeto. Cada participante ficou responsável de confeccionar uma postagem por semana, enquanto todos divulgariam essa postagem em suas redes sociais.

Assim, quanto à primeira semana de postagens após este processo decisório, ficou evidente que a maioria do público que respondeu possuía bons hábitos de higiene, levando à conclusão de que a população abordada estaria aberta ao processo de educação em saúde quanto à sífilis, dada a correlação entre bons hábitos de higiene e a autopercepção da saúde (Pinheiro, 2020). No entanto, alguns dados são notórios, como a higiene e troca de roupa da região íntima, pois uma higiene inadequada dos genitais pode propiciar o crescimento de microrganismos nocivos, além de facilitar a infecção por certas ISTs e parasitoses (Chen, Bruning, Rubino, \& Eder, 2017; Meza \& Salas, 2019). Além disso, há os hábitos de roer as unhas e de não lavar as mãos antes das refeições, podendo causar parasitoses e infecções gastrointestinais (Gallardo, 2017).

Em novembro, com o seguimento das postagens, alguns dos integrantes, principalmente na segunda e na terceira semanas, esqueceram de registrar as visualizações, que não puderam ser registradas, já que o aplicativo usado apaga as mesmas após $48 \mathrm{~h}$ da postagem. Assim, foram identificadas duas das dificuldades de utilizar redes sociais para atividades de extensão: os próprios integrantes às vezes desconheciam minúcias importantes do aplicativo, o que prejudicou o registro de dados; e a própria 
disciplina dos integrantes que, nesse projeto à distância, ficou mais fragilizada, fato este evidenciado pelo esquecimento no momento de registrar as visualizações. Essa desmotivação pode ser oriunda do próprio modelo virtual do projeto, como apontam Bizarria, Carneiro, Silva e Tassigny (2016), ao abordarem uma escala de avaliação de motivação na educação à distância.

Então, como houve um erro no registro das segunda e terceira semanas da segunda rodada de postagens, será abordado um panorama mais geral das visualizações. Dessa forma, a discussão sobre o motivo da média de visualizações por postagem decrescerem desde a postagem do panfleto é pertinente: houve um decréscimo de cerca de 837 visualizações por postagem a cerca de 413, na última semana de divulgações, ou seja, uma redução em torno de 49,3\%. De acordo com as postulações de Arigo, Pagoto, Carter-Harris, Lillie, e Nebeker (2018) sobre a falta de engajamento em pesquisas de saúde no Instagram ${ }^{\circledR}$ e no Facebook®, essa redução nas visualizações pode ter decorrido dos visuais do post (por exemplo: cores, imagens, linguajar); ou por ser um assunto mais estigmatizado, o que reduz a participação, fato provavelmente observado na ausência de perguntas quando era aberto o espaço para a tirada de dúvidas; por fim, o não uso de certas características que aumentam o alcance dos posts, como as "hashtags", também pode estar correlacionado. Por esse mesmo motivo, é possível supor que a rodada de postagens que mais recebeu visualizações, no total de 7.847, o fez por dispor de um método mais lúdico e bastante interativo para abordar a população, um questionário público simples e anônimo.

\section{Considerações Finais}

A Pandemia da COVID 19 desencadeou o surgimento de novos paradigmas para a extensão universitária, principalmente em relação a temas estigmatizados, como é o caso da Sífilis. Dessa forma, a comunidade acadêmica deve buscar formas de ultrapassar essas barreiras a fim de formar novas habilidades resolutivas sobre essa nova demanda social. Portanto, o presente projeto teve que ser adaptado e, apesar das dificuldades do novo formato, o mesmo atingiu um bom alcance, inclusive sendo divulgado nas plataformas online da Universidade Estadual do Piauí.

Ademais, diante do apresentado, conclui-se também que são necessários, futuramente, no momento em que tais medidas presenciais sejam permitidas, trabalhos de campo, utilizando medidas de testagem rápida, a fim de avaliar melhor a prevalência de ISTs na população, o que era o intuito inicial do projeto, antes da pandemia. Porém, diante dos resultados favoráveis observados neste relato de experiência, mais trabalhos tendo as redes sociais como metodologia principal também são recomendados, a fim de impactar uma grande quantidade de indivíduos, conscientizando-os sobre diversas condições negligenciadas na área da saúde.

\section{Referências}

Arigo, D., Pagoto, S., Carter-Harris, L., Lillie, S. E., \& Nebeker, C. (2018). Using social media for health research: Methodological and ethical considerations for recruitment and intervention delivery. Digital health, 4, 2055207618771757.

Ayres, J. R. D. C. M., Freitas, A. C., Santos, M. A. S. D., Saletti Filho, H. C., \& França Júnior, I. (2003). Adolescência e aids: avaliação de uma experiência de educação preventiva entre pares. Interface-Comunicação, Saúde, Educação, 7, 123-138.

Bizarria, F. P. A., Carneiro, T. C. J., Silva, M. A., \& Tassigny, M. M. (2016). Escala de Motivação Acadêmica: Validade no con texto da educação à distância em curso de administração pública. Revista Capital Científico-Eletrônica (RCC), e14(4), 75-91.

Chen, Y., Bruning, E., Rubino, J., \& Eder, S. E. (2017). Role of female intimate hygiene in vulvovaginal health: Global hygiene practices and product usage. Women's Health, 13(3), 58-67.

Chung, M. C. H. L., Marangon, M. B., Luna, W. F., Bettini, R. V., Watanabe, R. K., \& Shiroma, M. M. (2020). Desafios do Brincar com Idosos: Narrativas de Estudantes de Medicina do Programa Amigos do Sorriso. Revista Brasileira de Educação Médica, 44(4).

Dantas-Silva, L. T., Brito, V. C. N. G., Araújo, L. F., \& Macedo, J. Q. (2020). Práticas Educativas para Autocuidado Pós-Operatório Divulgadas por Meio de Mídia Digital. In Anais do V Congresso sobre Tecnologias na Educação, 492-499. https://doi.org/10.5753/ctrle.2020.11427

Decreto $n^{\circ} 19.531$, de 18 de março de 2020. Declara situação de Emergência em Saúde Pública no Município de Teresina, e dispõe sobre medidas de enfrentamento à pandemia provocada pelo novo Coronavírus (COVID-19), e dá outras providências. Teresina, 2020. https://pmt.pi.gov.br/wpcontent/uploads/sites/34/2020/03/decreto.pdf. 
Diniz, E. G. M., Silva, A. M., Nunes, P. H. V., Franca, W. W. M., Rocha, J. V. R., Silva, D. V. S. P., Santos, V. H. B., Araú jo, H. D. A., Albuquerque, M. C. P. A., \& Aires, A. L. (2020). A extensão universitária frente ao isolamento social imposto pela COVID-19. Brazilian Journal of Development, 6(9), 72999-73010.

Fernandes, P. (2020). \#Uespificaemcasa: Alunos do curso de Medicina realizam projeto de extensão sobre cuidados com a Sífilis. https://uespi.br/site/?p=135168.

Ferraz, I. S., \& Del Ciampo, L. A. (2019). Uma experiência exitosa de meio século da Universidade de São Paulo junto à comunidade. Medicina (Ribeirão Preto), 52(1), 1-6.

Gallardo, Z. J. C. (2017). Conocimiento Y Hábitos De Higiene Corporal Em Estudiantes Del V Ciclo Del Colegio De Aplicación Marcos Duran Martel Huánuco, 2016. Monografia de bacharelado, Facultad De Ciencias De La Salud, Programa Académico De Enfermería, Universidad de Huánuco, Huánuco, Peru.

Graphpad. QuickCalcs: Run statistical analyses quickly and directly in your browser. 2021. Recuperado em 08 de janeiro, 2021, de https://www.graphpad.com/quickcalcs/.

Luna, W. F., Nordi, A. B. D. A., Rached, K. S., Correia, M. B. A., Carvalho, A. R. V. D., \& Morais, L. F. D. D. (2020). Estudantes de Medicina em Roda: os Diálogos da Extensão Popular com os Indígenas Potiguara. Revista Brasileira de Educação Médica, 44(3).

Meza, A. S. C., \& Salas, J. L. P. (2019). Factores personales presentes en mujeres con síndrome de flujo vaginal atendidas en la estrategia ITS/VIH del Hospital Goyeneche en los meses de diciembre 2018 a enero 2019. Monografia de bacharelado, Facultad de Obstetricia y Puericultura, Escuela Profesional de Obstetricia y Puericultura, Universidad Católica de Santa María, Arequipa, Peru.

Ministério da Saúde. Boletim Epidemiológico: sífilis 2018. (2018). http://www.aids.gov.br/pt-br/pub/2018/boletim-epidemiologico-de-sifilis-2018.

Ministério da Saúde. Sífilis. (2017). http://portalms.saude.gov.br/saude-de-a-z/sifilis-2.

Moraes, S. L. D. D., Tamaki, R., Sobral, A. P. V., Júnior, J. F. S., Leão, R. D. S., Silva, B. G. D., \& Gomes, J. M. D. L. (2016). Impac to de uma experiência extensionista na formação universitária. Revista de Cirurgia e Traumatologia Buco-maxilo-facial, 16(1), 39-44.

Moura, L. D. F. A. D. D., Piauilino, R. J. B., Araújo, Í. F., Moura, M. S. D., Lima, C. C. B., Evangelista, L. D. M., \& Lima, M. D. D. M. D. (2012). Impacto de um projeto de extensão universitária na formação profissional de egressos de uma universidade pública. Revista de Odontologia da UNESP, 41(5), $348-352$.

Oliveira, F. L. B., \& Almeida Júnior, J. J. (2015). Extensão universitária: contribuições na formação de discentes de enfermagem. Revista Brasileira de Pesquisa em Saúde/brazilian Journal Of Health Research, 41(5), 348-352.

Peeling, R., Mabey, D., Kamb, M., Chen, X., Radolf, J. D., \& Benzaken, A. S. Syphilis. Nat Rev Dis Primers, 3, 1-49. https://doi.org/10.1038/nrdp.2017.73.

Pinheiro, D.B. (2020). Higiene pessoal: cuidando da saúde. In Da Paz, J.F. Poéticas Do Educar. 1, 99-114. Santa Catarina: Clube de Autores Publicações S/A.

Pinotti, C. (2020). University extension: setting and funding. Research, Society and Development, 9(5), e89953150. https://doi.org/10.33448/rsd-v9i5.3150.

Pinto, V. M., Basso, C. R., Barros, C. R. D. S., \& Gutierrez, E. B. (2018). Fatores associados às infecções sexualmente transmissíveis: inquérito populacional no município de São Paulo, Brasil. Ciência \& Saúde Coletiva, 23, 2423-2432.

Resende, J. C., Alves, R. B. D. S., Coutinho, M. D. S., Bragagnoli, G., \& Araújo, C. R. F. D. (2013). Importância da in iciação científica e projetos de extensão para graduação em medicina. Rev bras ciênc saúde [Internet], 17(1), 11-8.

Rocha, C. R., Moreira, A. P. A., Silva, L. R., Santos, I. M. M., Barbosa, M. N., Bittencourt, G., \& Feitosa, I. B. (2020). A utilização das redes sociais como estratégia para continuidade da extensão universitária em tempos de pandemia. Raízes e rumos, 8(1), 261-269.

Rodrigues, L. K. N., Freitas, M. D. S., \& Henriques A. C. P. T. Produção de conteúdo digital para abordagem do cuidado clínico cardiovascular: um relato de experiência. (2020). Conexão UNIFAMETRO 2020. https://doity.com.br/media/doity/submissoes/artigo-f0332da2c6db389168ee1a45d2eabalfd6ebad69segundo_arquivo.pdf.

Rowley, J., Hoorn, S. V., Korenromp, E., Low, N., Unemo, M., Abu-Raddad, L. J., Chico, R. M., Smolak, A., Newman, L., Gottlieb, S., Thwin, S. S., Broutet, N, \& Taylor, M. M. (2019). Chlamydia, gonorrhoea, trichomoniasis and syphilis: global prevalence and incidence estimates, 2016. Bulletin of the World Health Organization, 97(8), 548.

Silva, M. R. F., Mascarenhas, A. L. L. D., Silva, M. D. C. F., Dutra, G., Silva, C. A. F., \& Dias, N. S. (2020). Reflexões sobre as ações extensionistas e de pesquisa no combate à COVID-19 na universidade do estado do Rio Grande do Norte. Brazilian Journal of Health Review, 3(2), 3622-3646. 